\title{
Stationary pattern formation in a discrete excitable system with strong inhibitory coupling
}

\author{
Naoko Kurata, ${ }^{1}$ Hiroyuki Kitahata, ${ }^{1}$ Hitoshi Mahara, ${ }^{2}$ Atsushi Nomura, ${ }^{3}$ Hidetoshi Miike, ${ }^{4}$ and Tatsunari Sakurai ${ }^{1}$ \\ ${ }^{1}$ Graduate School of Science, Chiba University, Chiba 263-8522, Japan \\ ${ }^{2}$ Nanotechnology Research Institute, National Institute of Advanced Industrial Science and Technology (AIST), Tsukuba 305-8565, Japan \\ ${ }^{3}$ Faculty of Education, Yamaguchi University, Yamaguchi 753-8513, Japan \\ ${ }^{4}$ Faculty of Engineering, Yamaguchi University, Ube 755-8611, Japan
}

(Received 25 December 2008; published 5 May 2009)

\begin{abstract}
We study a discrete model described by coupled excitable elements following the monostable FitzHughNagumo equations. Our model has a weakly coupled activator and a strongly coupled inhibitor. For twocoupled excitable elements, we show that the trivial state always exists stably, while nontrivial stable states appear depending on the coupling strengths. In a one-dimensional array, only the elements near the initial condition step remain at nontrivial states. We discuss stationary pattern formation in a one-dimensional array and a two-dimensional lattice using the analytical results of a two-coupled system.
\end{abstract}

DOI: 10.1103/PhysRevE.79.056203

PACS number(s): 05.45.Xt, 45.70.Qj, 47.54.-r, 82.40.Bj

\section{INTRODUCTION}

Discrete systems are widely seen in living things. For example, myelinated fibers are constituted of myelin sheaths and nodes of Ranvier. Electric potential propagates from one node to another and this phenomenon is called saltatory conduction $[1,2]$. Not only the living phenomena but also nonliving ones, such as dislocation in crystals $[3,4]$, are described by models using spatial discreteness. Phenomena that occur in discrete systems can be quite different from those in continuous systems.

If the diffusion coefficient of the activator is larger than that of the inhibitor, pulses can propagate in continuous systems. However, in discrete systems, when the coupling strength of the activator is larger than that of the inhibitor, propagation can fail. Details of features in discrete models have been studied both analytically and numerically [5-9].

It is also well known that if the diffusion of the inhibitor is faster than that of the activator in continuous systems, a Turing pattern appears; pulses that appear from perturbation form patterns of stripes or spots [10-13]. In these patterns, the system's wave number depends on the parameters and the stripes or spots keep the same intervals regardless of the system size.

We are interested in what will happen in a discrete system under the same condition: a weakly coupled activator and a strongly coupled inhibitor. In such a system, Nomura et al. [14] reported that a stationary pattern appears. They claimed that they can detect the edge of an input image using a reaction-diffusion-like model. These patterns are quite different from the Turing pattern; the edge of the input image remains stable and steady and the pattern's characteristic wave number is not uniquely determined. This is also a different phenomenon from propagation failure. Propagation failure occurs in a discrete system; a wave can not propagate in a certain range of coupling strength [5-9]. Ebihara et al. [15] suggested that discreteness plays an important role in this phenomenon, but the details of discreteness have not been considered in their model.
In this study, we discuss the reason for the emergence of stationary patterns in a discrete system using two-coupled elements. We consider excitable elements in an activatorinhibitor system. They are coupled with each other under the condition of a weakly coupled activator and a strongly coupled inhibitor and follow the monostable FitzHughNagumo equations, which are well known as a simple example of the excitable systems $[16,17]$. Note that we consider a temporally continuous system. We analytically show that the trivial state is always stable in this system, but in a certain parameter area, other stable states appear. Which state the two elements finally reach depends on the initial conditions. If the initial conditions of both elements are larger (smaller) than a threshold, both elements reach the trivial steady state after (without) excitation. On the other hand, if the initial condition of one element is greater than the threshold, but that of the other is less than it, both elements enter a new stable steady state. This feature implies that when we adopt a two-dimensional lattice and input a certain image as an initial condition, the edge of the image can be detected using this feature.

\section{MODEL}

We use the following discrete model for two nonlinear excitable elements, which have a weakly coupled activator, $u$, and a strongly coupled inhibitor, $v$. The elements interact with each other depending on the coupling strength, $d_{u}$ and $d_{v}[18]$. The terms $f(u, v)$ and $g(u, v)$ are considered to represent the FitzHugh-Nagumo equations as follows:

$$
\begin{gathered}
\frac{d u_{i}}{d t}=d_{u}\left(u_{j}-u_{i}\right)+f\left(u_{i}, v_{i}\right), \\
\frac{d v_{i}}{d t}=d_{v}\left(v_{j}-v_{i}\right)+g\left(u_{i}, v_{i}\right), \\
(i, j=1,2, i \neq j)
\end{gathered}
$$



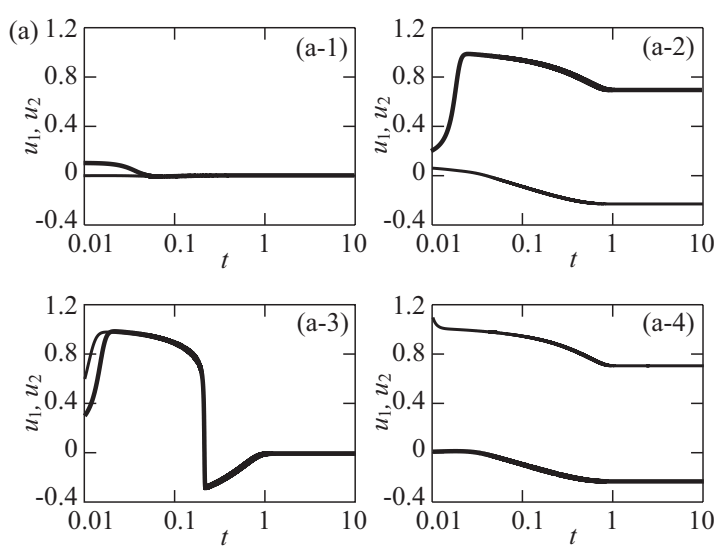

(b)

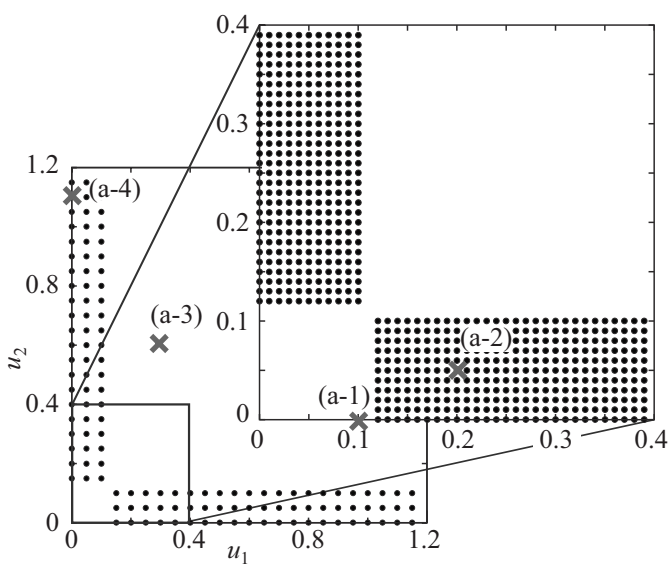

FIG. 1. (a) Time variation of $u_{1}$ and $u_{2}$. The horizontal axis is logarithmic. Bold lines and thin lines represent the time variation of $u_{1}$ and $u_{2}$, respectively. The initial values of $\left(u_{1}, u_{2}\right)$ are $(0.1,0)$, $(0.2,0.05),(0.3,0.6)$, and $(0,1.1)$. (b) Dependence on initial conditions of the two elements. Filled circles show that one element finally becomes stable at a positive value and the other at a negative value. Sets of the initial values of time variation in Fig. 1(a) correspond to the crosses.

$$
\begin{gathered}
f(u, v)=\frac{1}{\epsilon}\{-u(u-1)(u-a)-v\}, \\
g(u, v)=u-b v .
\end{gathered}
$$

The parameters, $\epsilon, a$, and $b$, are positive constants; in this study, they are set to $0.001,0.1$, and 2 , respectively. Under this condition, the model becomes a monostable system. Then we use the values of $d_{u}=1$ and $d_{v}=20$ without any notice.

\section{RESULTS}

We numerically investigate the dependence of the final states on the initial values of the two elements. In this part, we examine only the final states of $u_{1}$ and $u_{2}$ by changing their initial values. The initial values of $v_{1}$ and $v_{2}$ are set to 0 . Figure 1(a) shows the time variation under four types of initial conditions. In the first case, both elements converge to the trivial state without excitation. In the second (fourth) case, $u_{1}\left(u_{2}\right)$ becomes positive after excitation, and $u_{2}\left(u_{1}\right)$ becomes negative without excitation. In the third case, both elements go to the trivial state after excitation. We examine the details of the dependence by changing each initial value in $0 \leq u_{i} \leq 1.2(i=1,2)$, as shown in Fig. 1(b). Filled circles show that one value finally becomes negative and the other becomes positive. Empty areas represent cases where both elements finally become the trivial solution. Crosses represent the initial values in Fig. 1(a). The figure shows that there are thresholds around $u_{1}, u_{2}=0.11$.

Next, we want to analytically find the new state and investigate its stability. To find other solutions except the trivial solution, we set $U_{1}, U_{2}, V_{1}$, and $V_{2}$ as $u_{1}+u_{2}, u_{1}-u_{2}$, $v_{1}+v_{2}$, and $v_{1}-v_{2}$, respectively. Then we obtained the following equation from Eqs. (1) and (2):

$$
\begin{gathered}
\frac{d U_{1}}{d t}=\frac{1}{\epsilon}\left\{-\frac{U_{1}}{4}\left(U_{1}^{2}+3 U_{2}^{2}\right)+\frac{1}{2}(a+1)\left(U_{1}^{2}+U_{2}^{2}\right)-a U_{1}-V_{1}\right\}, \\
\frac{d V_{1}}{d t}=U_{1}-b V_{1}, \\
\frac{d U_{2}}{d t}=\frac{1}{\epsilon}\left\{-\frac{U_{2}}{4}\left(3 U_{1}^{2}+U_{2}^{2}\right)+(a+1) U_{1} U_{2}-a U_{2}-V_{2}\right\} \\
-2 d_{u} U_{2}, \\
\frac{d V_{2}}{d t}=U_{2}-b V_{2}-2 d_{v} V_{2} .
\end{gathered}
$$

To obtain the fixed points and their existence conditions from the equations, we calculate Eq. (3) without temporal change,

$$
\frac{d U_{1}}{d t}=\frac{d U_{2}}{d t}=\frac{d V_{1}}{d t}=\frac{d V_{2}}{d t}=0 .
$$

Equations (3) and (4) have the trivial solution, $\bar{U}_{1}=\bar{U}_{2}=\bar{V}_{1}$ $=\bar{V}_{2}=0$. As for the nontrivial solution, we can reduce the variables and get the following equation for $\bar{U}_{2}$ :

$$
\bar{U}_{2}^{2}=\frac{-\bar{U}_{1}\left\{\bar{U}_{1}^{2}-2(a+1) \bar{U}_{1}+4\left(a+\frac{1}{b}\right)\right\}}{3 \bar{U}_{1}-2(a+1)} \geq 0 .
$$

Here, for the requirement that the system is monostable, following equation

$$
u\left\{u^{2}-(a+1) u+\left(a+\frac{1}{b}\right)\right\}=0,
$$

which is obtained by Eq. (2), should have only one solution $u=0$. Then we get the condition,

$$
(a+1)^{2}-4\left(a+\frac{1}{b}\right)<0 .
$$

From this condition, the discriminant of $\left\{\bar{U}_{1}^{2}-2(a+1) \bar{U}_{1}\right.$ $+4(a+1 / b)\}$ in Eq. (5) is negative. As a result, we get the requirement about $U_{1}$, 

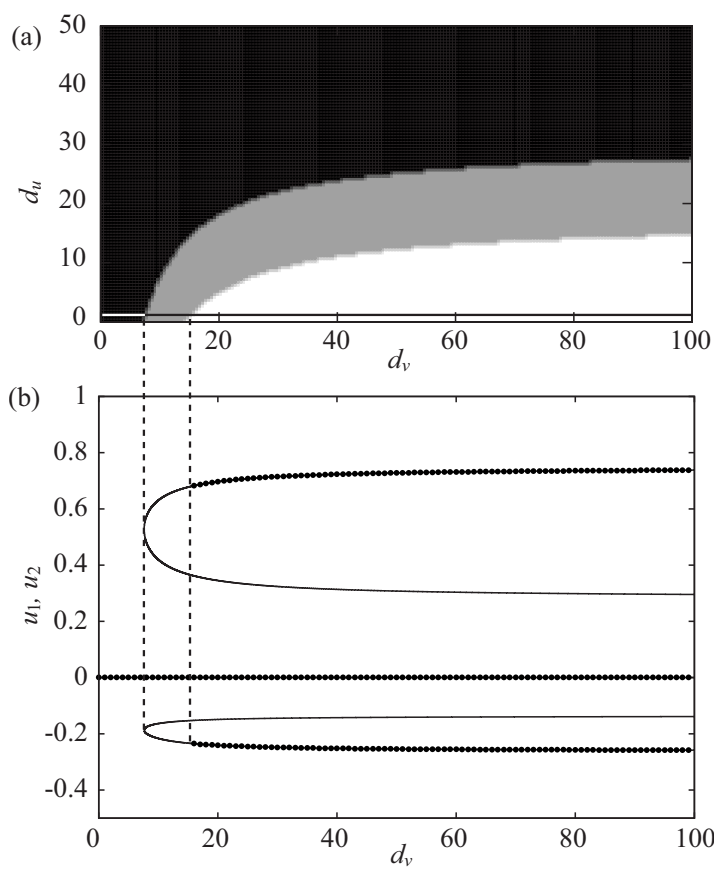

FIG. 2. (a) Parameter area of stability of the nontrivial solutions obtained by linear stability analysis. In the white, gray, and black regions, nontrivial solutions exist stably, exist unstably, and do not exist, respectively. (b) Solutions of Eqs. (3) and (4), when we fix $d_{u}=1$ and consider $d_{v}$ to be a bifurcation parameter along a line in (a). The longitudinal axis is the value of $u_{1}$ and $u_{2}$. Filled circles represent stable solutions achieved by numerical calculation; solid lines represent analytical solutions.

$$
0<\bar{U}_{1}<\frac{2}{3}(a+1)
$$

We can obtain following cubic equation for $\bar{U}_{1}$ by calculating Eqs. (3)-(5):

$$
\begin{aligned}
\left\{\bar{U}_{1}\right. & \left.-\frac{2}{3}(a+1)\right\}^{3}-\Xi\left\{\bar{U}_{1}-\frac{2}{3}(a+1)\right\}+(a+1) \\
& \times\left\{\frac{2 a^{2}-5 a+2}{27}-\frac{1}{3 b}\right\}=0, \\
\Xi & =\frac{1}{3}\left(a^{2}-a+1\right)+\frac{1}{2 b}-\frac{3}{2}\left(\frac{1}{b+2 d_{v}}+2 d_{u} \epsilon\right) .
\end{aligned}
$$

Equations (3) and (4) can have the other solutions except for zero when cubic Eq. (5) has real solutions in the condition Eq. (8). Through analytical calculations, the condition, where Eqs. (3) and (4) have nontrivial solutions, is obtained as

$$
\begin{aligned}
\frac{1}{b+2 d_{v}}+2 d_{u} \epsilon \leq & \frac{2}{9}\left(a^{2}-a+1\right) \\
& +\frac{1}{3 b}-\frac{2}{3}\left[\frac{1+a}{6 \sqrt{3}}\left(\frac{9}{b}-2 a^{2}+5 a-2\right)\right]^{2 / 3} .
\end{aligned}
$$

The derived condition is represented by the boundary between the black and gray areas in Fig. 2(a).
From the linear stability analysis, we can conclude that the trivial solution is always stable, and the stability of nontrivial solutions is represented by the gray and white areas in Fig. 2(a). In the gray area, nontrivial solutions exist, but they are unstable. In the white area, nontrivial solutions exist stably. Figure 2(b) shows the analytical solutions of Eqs. (3) and (4) obtained by solving the cubic equation, Eqs. (9) and (10). The value of $d_{u}$ is fixed as 1 , and $d_{v}$ changes from 0 to 100 along the axis in Fig. 2(a). Filled circles superimposed on Fig. 2(b) represent stable solutions obtained by numerical calculations. Note that numerical calculations started from an initial condition that was determined by the final state at a neighboring parameter with an increase (decrease) in $d_{u}$.

Next, we consider more than two elements. The model is as follows:

$$
\begin{aligned}
& \frac{d u_{i}}{d t}=d_{u}\left(u_{i+1}-u_{i}\right)+d_{u}\left(u_{i-1}-u_{i}\right)+f\left(u_{i}, v_{i}\right), \\
& \frac{d v_{i}}{d t}=d_{v}\left(v_{i+1}-v_{i}\right)+d_{v}\left(v_{i-1}-v_{i}\right)+g\left(u_{i}, v_{i}\right),
\end{aligned}
$$

$$
(i=1,2, \ldots, N) \text {. }
$$

Each is coupled with its neighbors. Actually, this is equivalent to the discretized reaction-diffusion equation by using the central difference method with zero flux boundary condition. For example, we use $u_{0}, u_{1}, \ldots, u_{N+1}$ and consider $u_{0}=u_{1}$ and $u_{N}=u_{N+1}$ at the boundary. Figures 3(a)-3(c) show the behavior of three elements $(N=3)$. When we set the initial value of $u_{3}$ as 0.15 and the other values as 0 , the final value of $u_{3}$ becomes positive after excitation, and the others become negative without excitation [Fig. 3(a)]. In this case, the value of $u_{2}$ is a little smaller than that of $u_{1}$. When $u_{2}$ is 0.15 and the others are zero, only $u_{2}$ excites and goes to a positive value, while the others go to the same negative value [Fig. 3(b)]. If we set the initial value on elements 2 and 3 as $u_{2}=u_{3}=0.15$ and $u_{1}=0$, elements 2 and 3 both excite at first, but then element 3 falls to a negative value [Fig. 3(c)]. In the three-element system, emergence of a nontrivial stable state, negative value, and positive value is confirmed in each gray parameter area, as shown in (a-1)-(c-1) of Figs. 3(a)-3(c). The shape of parameter areas (a-1) and (b-1) is similar to those of the two-element system, as shown in Fig. 2(a). The result shown in Fig. 3(c) illustrates that its parameter area is smaller than those in (a-1) and (b-1).

Figure $3(\mathrm{~d})$ shows the time variation of a one-dimensional array of 16 excitable elements when a step function is provided as an initial condition. In Fig. 3(d), all elements to which a small signal is added (d-1) are excited (d-2). The elements except those at the edge fall to negative values (d-3), and then only the edge remains stable and steady (d-4).

\section{DISCUSSION}

In this study, we discussed stationary pattern formation on coupled nonlinear excitable elements following the monostable FitzHugh-Nagumo model using two-coupled elements. From the analysis, we can determine the parameter 


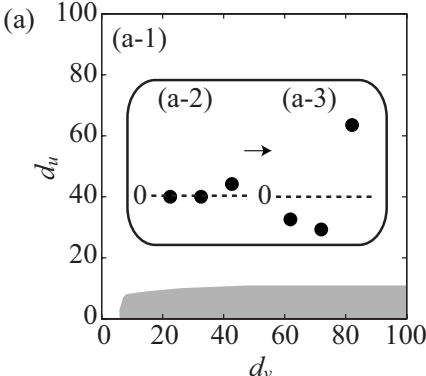

(d)

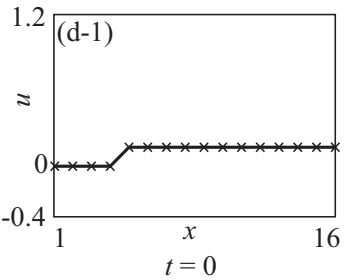

(b)
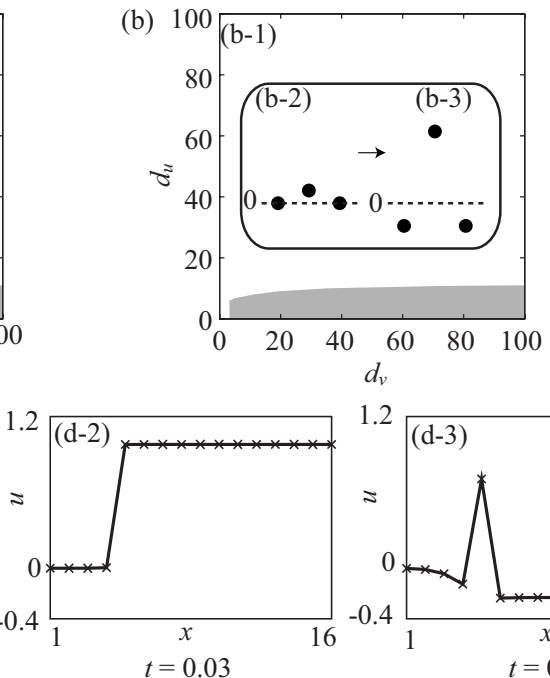

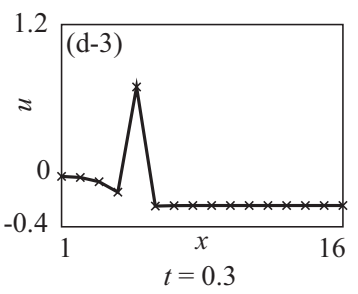

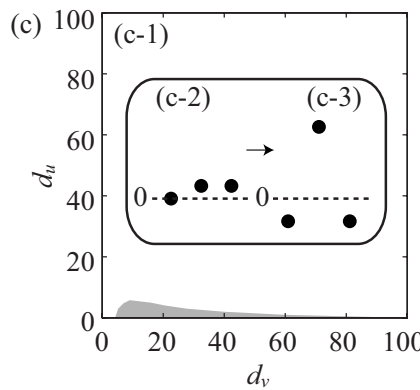

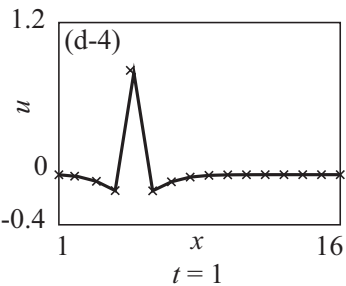

FIG. 3. (a)-(c) Behavior of three coupled elements under three types of initial conditions, (a-2), (b-2), and (c-2), respectively. In the empty areas shown in (a-1), (b-1), and (c-1), all elements finally reach the trivial state. In the gray areas, elements reach the states shown in (a-3), (b-3), and (c-3). (d) Time variation of a one-dimensional array. In this case, each of sixteen elements is coupled with its two neighbors. The horizontal axis represents space, and the longitudinal axis is the value of $u$. Initial values are provided as (d-1). Elements with a larger initial value excite (d-2) and fall to negative values (d-3). After sufficient time, the edge of the step function remains and other elements except ones around the edge go to the trivial state, zero (d-4). Parameters are $d_{u}=1$ and $d_{v}=4$. It is confirmed numerically that the situation of elements at $t=1$ keeps its shape until at least $t=10$.

area in which two stable nontrivial solutions appear except for the trivial solution, zero. The analytical work also shows that the trivial solution constantly exists stably. The initial conditions determine whether the final state of both elements is trivial or nontrivial. The emergence of not only the trivial stable state, but also the nontrivial stable state in a twoelement system implies the existence of those states in a three-element system, a one-dimensional system, and a twodimensional system. This is confirmed numerically in a three-element system and a one-dimensional system, as shown in Fig. 3.

For application of two-coupled elements, when we consider a two-dimensional lattice of excitable elements, each coupled with four neighbors, a stationary pattern can also

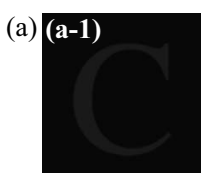

$t=0$

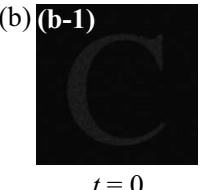

$t=0$

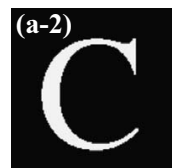

$t=0.04$

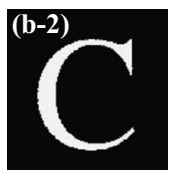

$t=0.04$

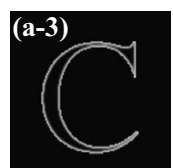

$t=0.21$

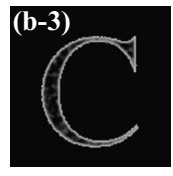

$t=0.21$

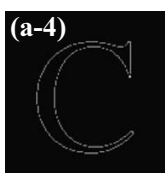

$t=1$

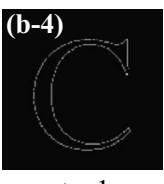

$t=1$
FIG. 4. (a),(b) Time variation of the two-dimensional image. (a-1) Initial image where $u=0.15$ in the gray area, and $u=0$ elsewhere. (a-2) Elements that are given the initial value $u=0.15$ all excite. (a-4) Finally the edge of the initial image remains. (b) Initial image contains a white noise between \pm 0.04 in the entire area of (a-1). Parameters are $d_{u}=1$ and $d_{v}=4$. It is confirmed numerically that the situation of elements at $t=1$ keeps its shape until at least $t=10$. emerge, as shown in Fig. 4. We set an initial condition as in (a-1) of Fig. 4(a); in the black area, the initial value of $u$ is zero, and in the gray area, shaped like the letter "C," each element has a low value of $u, 0.15$. Note that setting the initial value of $u$ for two-dimensionally coupled elements is equivalent to putting an image on the lattice. Elements that are given the initial value of $u=0.15$ excite, as shown in (a-2) of Fig. 4(a). In the middle of the white area, where the difference in $u$ between an element and its neighbors is small, they converge to zero, i.e., the black area in the middle of a white region in (a-3) of Fig. 4(a). Then only the edge remains at positive values and a stationary pattern appears, as shown in (a-4) of Fig. 4(a). When the initial image has white noise, the noise is reduced in the final state, as shown in Fig. 4(b). In this case, the threshold plays an important role in reducing the effect of weak noise. The emergence of stationary patterns in a spatially discrete system can demonstrate wide flexibility in image processing. Recently, patterns in Belousov-Zhabotinsky (BZ) reaction in aerosol OT (AOT) water-in-oil microemulsion are studied [19], and some patterns which are different from those in normal BZ reaction are observed such as Turing-like patterns, dashed waves, and so on. Microemulsions of water are discretized by oil, so this system may be described as a discrete system and the diffusion of inhibitor is faster than that of activator. These imply that our model may be experimentally actualized by using a Ru-catalyzed BZ-AOT system.

\section{ACKNOWLEDGMENTS}

The authors are deeply indebted to Dr. Masaharu Nagayama for his invaluable discussion. This work was supported in part by Grants-in-Aid for Young Scientists A (Grant No. 18684022) (T.S.). 
[1] J. P. Keener and J. Sneyd, Mathematical Physiology (Springer, New York, 1998).

[2] A. C. Guyton and J. E. Hall, Textbook of Medical Physiology (Saunders, Philadelphia, 2006).

[3] L. L. Bonilla and A. Carpio, Physica A 376, 361 (2007).

[4] A. Carpio and L. L. Bonilla, Phys. Rev. Lett. 90, 135502 (2003).

[5] J. P. Keener, SIAM J. Appl. Math. 47, 556 (1987).

[6] V. Booth and T. Erneux, Physica A 188, 206 (1992).

[7] Y. Nishiura, D. Ueyama, and T. Yanagita, SIAM J. Appl. Dyn. Syst. 4, 733 (2005).

[8] A. Carpio, L. L. Bonilla and A. Luzón, Phys. Rev. E 65, 035207(R) (2002).

[9] A. Carpio and L. L. Bonilla, SIAM J. Appl. Math. 63, 619 (2002).

[10] A. M. Turing, Philos. Trans. R. Soc. London, Ser. B 237, 37
(1952).

[11] Q. Ouyang and H. L. Swinney, Nature (London) 352, 610 (1991).

[12] A. Nomura, M. H. Miike, T. Sakurai, and E. Yokoyama, J. Phys. Soc. Jpn. 66, 598 (1997).

[13] J. E. Pearson, Science 261, 189 (1993).

[14] A. Nomura, M. Ichikawa, H. Miike, M. Ebihara, H. Mahara, and T. Sakurai, J. Phys. Soc. Jpn. 72, 2385 (2003).

[15] M. Ebihara, H. Mahara, T. Sakurai, A. Nomura, and H. Miike, Proceedings of Visualization, Imaging, and Image Processing (Acta, Calgary, 2003), Vol. 396, p. 145.

[16] R. FitzHugh, Biophys. J. 1, 445 (1961).

[17] J. Nagumo, S. Arimoto, and S. Yoshizawa, Proc. IRE 50, 2061 (1962).

[18] J. Miyazaki and S. Kinoshita, Phys. Rev. E 74, 056209 (2006).

[19] V. K. Vanag and I. R. Epstein, Chaos 18, 026107 (2008). 